\title{
Status of single phase and dual phase DUNE prototype detectors at CERN
}

\author{
Leigh H. Whitehead ${ }^{* \dagger}$ \\ CERN \\ E-mail: leigh.howard.whiteheadecern.ch
}

DUNE is a next-generation long-baseline neutrino oscillation experiment designed to measure the neutrino mass hierarchy and CP-violation in the lepton sector. ProtoDUNE-SP and ProtoDUNEDP are prototype detectors for the DUNE experiment, testing the single-phase and dual-phase liquid argon time projection chamber technologies, respectively. The detectors represent a key step towards realising the full $10 \mathrm{kt}$ liquid argon time projection chamber detector modules that will form the far detector of DUNE. The status of the two ProtoDUNE detectors and the future timescales of the experiments are discussed.

EPS-HEP 2017, European Physical Society conference on High Energy Physics 5-12 July 2017

Venice, Italy

* Speaker.

${ }^{\dagger}$ On behalf of the DUNE Collaboration 


\section{Introduction}

DUNE is a next-generation long-baseline neutrino oscillation experiment due to start atmospheric neutrino data collection in 2024 and neutrino beam data collection from 2026 [1]. Neutrinos produced by a high-power beam originating from Fermilab, USA, will travel $1300 \mathrm{~km}$ to the Sanford Underground Research Facility in South Dakota, USA. The neutrinos will be detected by four very large liquid argon time projection chambers (LArTPCs), each containing $10 \mathrm{kton}$ of liquid argon. The detectors will be located approximately $1.5 \mathrm{~km}$ underground to minimise the backgrounds from cosmic rays. Two different LArTPC technologies are under consideration for DUNE: single-phase (SP) and dual-phase (DP). The single-phase LArTPC design contains only liquid argon and it is the technology that will be used for the first of the DUNE far detector modules. The dual-phase detector technology uses a layer of argon gas above the liquid argon as an electron multiplier to amplify the charge liberated by traversing charged particles.

These 10 kton LArTPCs will be by far the largest ever built - for comparison the largest running LArTPC was ICARUS T600 that had two 300 ton modules [2]. A critical stage on the research and development path towards the final DUNE detectors is the construction of the two DUNE prototype detectors, ProtoDUNE-SP and ProtoDUNE-DP, in a purpose-built facility at CERN, Switzerland. They will use full-size components identical to those planned for the DUNE detectors to test the designs, engineering solutions, installation procedures, and the long-term performance and stability of the detector technologies. Each of the ProtoDUNE detectors will contain $800 \mathrm{t}$ of liquid argon making them the largest LArTPCs ever built in their own right. The new facility in the North Area at CERN includes two new very low energy extensions to the existing $\mathrm{H} 2$ and $\mathrm{H} 4$ beam-lines that will provide mixed charged particle beams $\left(e^{ \pm}, \mu^{ \pm}, \pi^{ \pm}, p\right.$, and a small fraction of $\left.K^{ \pm}\right)$in the momentum range $0.5 \mathrm{GeV} / \mathrm{c}$ to $7.0 \mathrm{GeV} / \mathrm{c}[3,4]$.
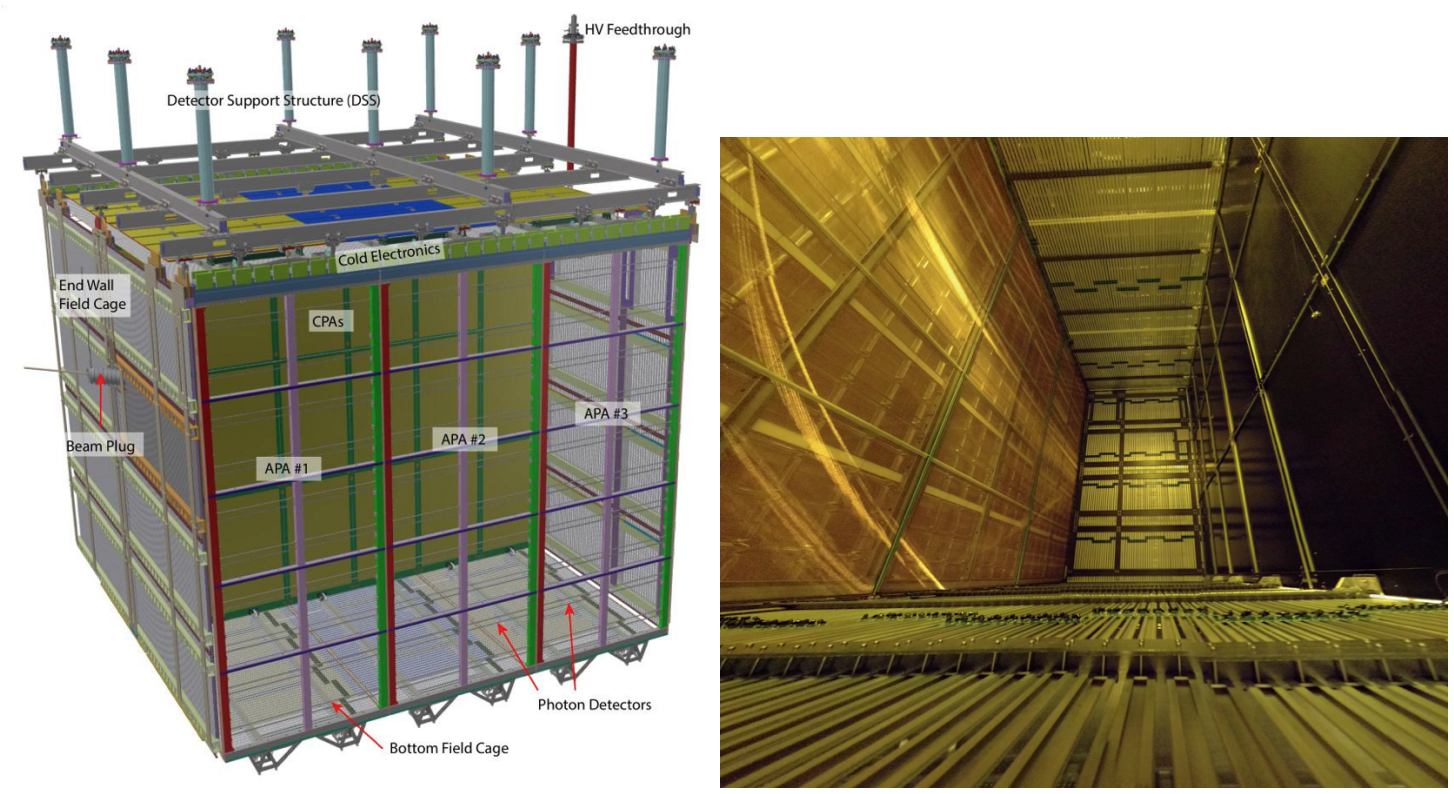

Figure 1: Schematic image of ProtoDUNE-SP (left [4]) and a photograph of a completed drift volume (right). 


\section{ProtoDUNE-SP}

ProtoDUNE-SP, shown schematically on the left of Fig. 1, has two $3.2 \mathrm{~m}$ long drift volumes separated by a central cathode biased to a voltage of $-180 \mathrm{kV}$. The charge readout is performed by six anode plane assemblies (APAs); each APA consists of three wire planes. Charged particles traverse the liquid argon and deposit energy through ionisation and the ionisation electrons drift in an electric field of $0.5 \mathrm{kVcm}^{-1}$ towards the APAs. The electrons drift past the first two wire planes and induce charge in the wires before being collected by the third and final wire plane, giving three independent views of each interaction. The total active volume measures $7.2 \times 6.0 \times 7.0 \mathrm{~m}^{3}$.

Four of the six APAs were constructed at PSL in the US and the remaining two were constructed at Daresbury Laboratory in the UK. Each of the APAs with their attached cold electronics were tested in a cold box located next to the detector cryostat on their arrival at CERN. The cold box was filled with gaseous nitrogen at $150 \mathrm{~K}$ to test the complete APAs in conditions similar to the detector without using liquid argon.

As of $6^{\text {th }}$ July 2018 all of the detector components were fully installed, as demonstrated by the photograph of one of the completed drift volumes on the right of Fig. 1. The image shows the central cathode on the right, three of the APAs on the left and part of the field cage in the centre. The detector was hence ready to be purged with cold argon gas before it was filled with liquid argon. The detector will be exposed to a mixed charged particle beam in autumn 2018 and collect cosmic muon data throughout 2019.

\section{ProtoDUNE-DP}

ProtoDUNE-DP has a single $6.0 \times 6.0 \times 6.0 \mathrm{~m}^{3}$ drift volume with the cathode grid at the bottom of the detector, as shown in the left panel of Fig. 2. The ionisation electrons drift vertically in the $0.5 \mathrm{kVcm}^{-1}$ electric field towards the charge readout plane (CRP). The CRP provides the extraction of the ionisation electrons from the liquid phase to the gas phase, the charge amplification and the collection of the charge. The charge is extracted from the liquid with a $2 \mathrm{kVcm}^{-1}$ electric field, drawing the charge into the large electron multiplier (LEM) that amplifies the charge before it is collected by a PCB anode with two independent readout views.

As of $6^{\text {th }}$ July 2018 the first of the CRP modules, $3 \mathrm{~m} \times 3 \mathrm{~m}$ in size, had been completed and placed into a cold box containing liquid argon to begin testing, as shown on the right of Fig. 2. The installation of the detector components is foreseen to be completed by the end of 2018 with detector operation due to begin in 2019.

\section{Data and Analysis}

Whilst the ProtoDUNEs are primarily engineering prototypes, they provide an excellent testbed for the detector response, calibrations, event reconstruction software and analyses. The $\frac{d E}{d x}$ will be measured for the charged particles from the beam and cosmic muons, an important tool for measuring the energy scale and particle identification in DUNE. A long term goal of the event reconstruction software is the demonstration of $\pi^{0}$ reconstruction through the identification and measurement of the two decay photons. The primary physics goal of ProtoDUNE-SP is the measurement of the $\pi^{ \pm}$cross section on liquid argon, following the technique developed by LArIAT [5]. 

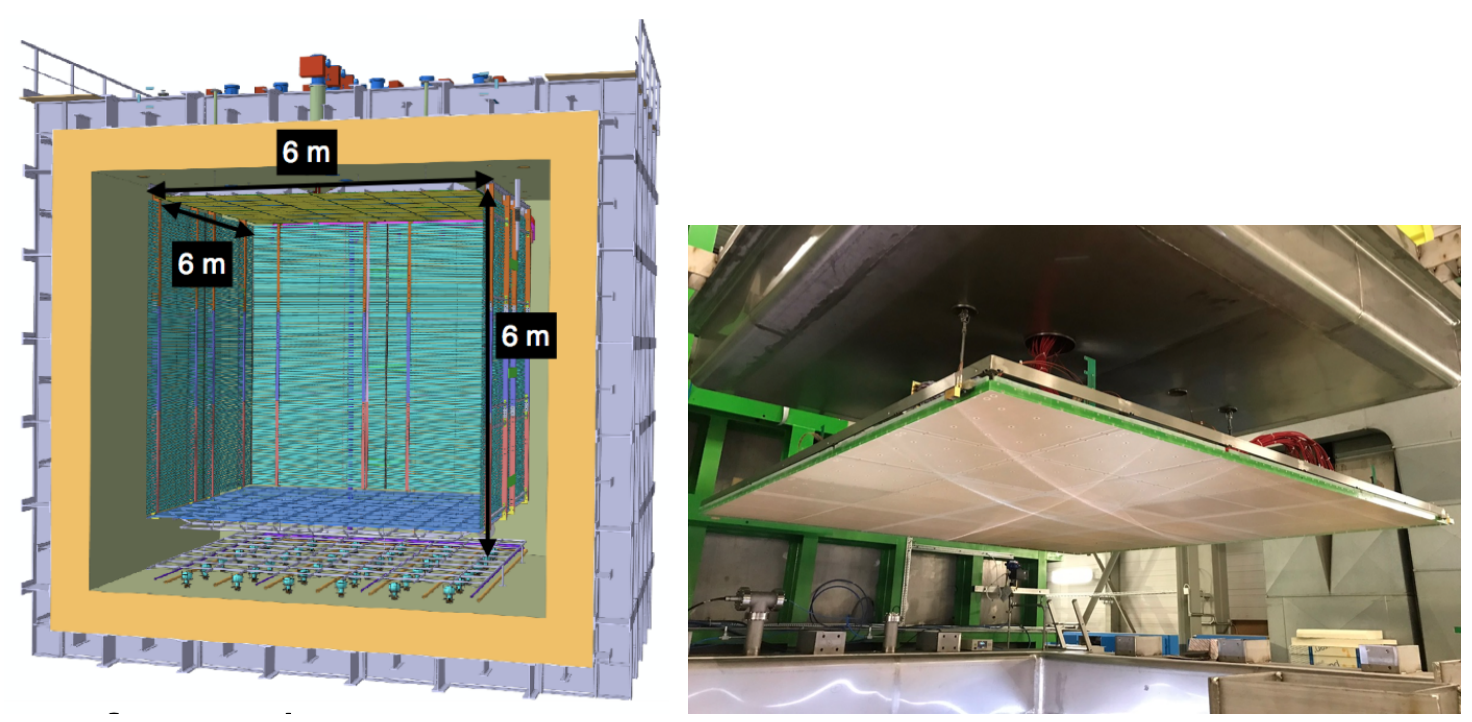

Figure 2: Schematic image of ProtoDUNE-DP (left) and a photograph of a completed CRP being lowered into the cold box (right).

The long run period during 2019 provides an opportunity for rare-event searches in the cosmic frontier such as the proposed search for inelastic boosted dark matter [6]. Studies and measurements from ProtoDUNE will provide important inputs to the DUNE technical design report, simulation improvements and the minimisation of systematic uncertainties.

\section{Summary}

The two ProtoDUNE detectors are under construction at CERN with the single-phase detector set to begin operation in autumn 2018. The dual-phase detector will begin to collect data at the start of 2019. The detectors will validate and test the designs of the DUNE far detector modules as well as provide an opportunity for both low-level and high-level physics measurements.

\section{References}

[1] R. Acciari et al. (DUNE), Long-Baseline Neutrino Facility (LBNF) and Deep Underground Neutrino Experiment (DUNE), arXiv 1601.05471, 2016.

[2] F. Arneodo et al. (ICARUS), The ICARUS liquid argon time projection chamber, Nucl. Instrum. Meth. A471 272-275, 2000.

[3] F. Tellander and N. Charitonidis, Tertiary particle production and target optimization of the $\mathrm{H} 2$ beam line in the SPS North Area, arXiv 1610.09066, 2016.

[4] B. Abi et al. (DUNE), The Single-Phase ProtoDUNE Technical Design Report, arXiv 1706.07081, 2017.

[5] J. Hugon (LArIAT), Pion scattering with LArIAT experiment, PoS NuFact17 069, 2017.

[6] A. Chatterjee et al., Search for Boosted Dark Matter at ProtoDUNE, Phys. Rev. D 98, 075027, 2018. 